\title{
Dwell time-based stabilisation of switched delay systems using free-weighting matrices
}

\section{Ahmet Taha Koru, Akın Delibaşı \& Hitay Özbay}

To cite this article: Ahmet Taha Koru, Akın Delibașı \& Hitay Özbay (2018) Dwell time-based stabilisation of switched delay systems using free-weighting matrices, International Journal of Control, 91:1, 1-11, DOI: 10.1080/00207179.2016.1266515

To link to this article: https://doi.org/10.1080/00207179.2016.1266515

Accepted author version posted online: 01

Dec 2016.

Published online: 20 Jan 2017.

Submit your article to this journal $\pi$

III Article views: 340

View Crossmark data

4 Citing articles: 4 View citing articles 준 


\title{
Dwell time-based stabilisation of switched delay systems using free-weighting matrices
}

\author{
Ahmet Taha Koru $\odot^{a}$, Akın Delibaşı ${ }^{b}$ and Hitay Özbay ${ }^{c}$ \\ ${ }^{a}$ Department of Mechatronics Engineering, Yıldız Technical University, İstanbul, Turkey; ${ }^{b}$ Department of Control and Automation Engineering,

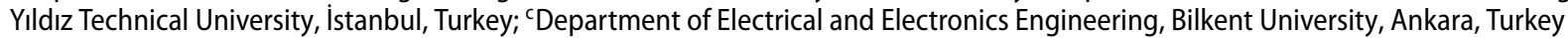

\begin{abstract}
In this paper, we present a quasi-convex optimisation method to minimise an upper bound of the dwell time for stability of switched delay systems. Piecewise Lyapunov-Krasovskii functionals are introduced and the upper bound for the derivative of Lyapunov functionals is estimated by freeweighting matrices method to investigate non-switching stability of each candidate subsystems. Then, a sufficient condition for the dwell time is derived to guarantee the asymptotic stability of the switched delay system. Once these conditions are represented by a set of linear matrix inequalities, dwell time optimisation problem can be formulated as a standard quasi-convex optimisation problem. Numerical examples are given to illustrate the improvements over previously obtained dwell time bounds. Using the results obtained in the stability case, we present a nonlinear minimisation algorithm to synthesise the dwell time minimiser controllers. The algorithm solves the problem with successive linearisation of nonlinear conditions.
\end{abstract}

\section{ARTICLE HISTORY}

Received 23 March 2016

Accepted 26 November 2016

\section{KEYWORDS}

Time-delay; dwell time optimisation; switched delay systems; free-weighting matrices

\section{Introduction}

A switched system is a dynamical system that includes a set of subsystems and a discrete switching event between them. General behaviour of a switched system is governed by following differential equation:

$$
\dot{x}(t)=f_{\sigma(t)}(x(t)), \quad \forall t>t_{0},
$$

where $\sigma$ denotes the switching signal which is a piecewise constant map from time to an index set representing subsystems. See the survey of Lin and Antsaklis (2009) for a review of the recent results and further references.

The stability analysis encountered in switched systems can be classified into three categories (Mahmoud, 2010). The first one is to find common Lyapunov functions so that the switched systems are stable under any arbitrary switching signal (Fainshil, Margaliot, \& Chigansky, 2009; Hou, Fu, \& Duan, 2013; Shorten, Narendra, \& Mason, 2003). The second one is to construct certain switching signals that make the switched system asymptotically stable (Liberzon \& Morse, 1999). The third category is the slow switching strategies such as dwell time stability or average dwell time stability for which the system is asymptotically stable (Geromel \& Colaneri, 2006; Hespanha, 2004; Hespanha \& Morse, 1999; Mitra \& Liberzon, 2004; Zhang, Han, Zhu, \& Huang, 2013). The class of switching signals can be restricted to signals with the property that the interval between any consecutive switching times is not less than a value called the dwell time. The switched delay system is asymptotically stable if all of the candidate subsystems are asymptotically stable and the dwell time is large enough (Morse, 1996). Most switched systems do not share a common Lyapunov function (Chen \& Zheng, 2010). Furthermore, having a common Lyapunov function is a sufficient condition for the stability under arbitrary switching, so it can be found conservative (Lin \& Antsaklis, 2009).

In this paper, we present some results on the dwell time stability analysis and stabilisation of the switched delay systems. A dwell time is observed in many switching system applications. The time intervals between the change in the road conditions among dry, wet and dirt for a car on the road can be considered as an example (Allerhand \& Shaked, 2011). Also, the slow switching strategies with dwell time can avoid chattering problems which can damage the physical systems (Ishii \& Francis, 2001). As a result, the stability analysis and stabilisation of switched systems with dwell time are increasingly popular.

The literature is abounded with various approaches for the stability analysis of time-delay systems, one can refer to $\mathrm{Gu}$, Kharitonov, and Chen (2003) for a review on the topic. Common methods to deal with delay-dependent stability problems are model transformations. In this method, point wise delay system transferred into a dis- 
tributed delay system. Stability of the transformed system is a sufficient condition for the stability of the original system. Hence, stability analysis with model transformations leads to a sort of conservatism since analysis operates on the transformed system instead of the original system $(\mathrm{Gu}$ et al., 2003). A less conservative approach to stability anal$y$ sis is the free-weighting matrices method which does not include any model transformation of the original system (He, Wang, Xie, \& Lin, 2007; Mahmoud, 2010; Wu, He, \& She, 2010). In this paper, we present some results for switched delay systems with pointwise delays.

There are recent results on dwell time stability of the switched delay systems. In Sun, Zhao, and Hill (2006) and Li, Gao, Agarwal, and Kaynak (2013), stability conditions are presented for a given average dwell time. In those papers, the conditions involve exponential and bilinear terms when the dwell time is considered as a free parameter. Hence, the minimisation of the dwell time and synthesising the dwell time minimiser controllers with those methods are not tractable. There are some optimisationbased methods to minimise the upper bound for the dwell time (Çalışkan, Özbay, \& Niculescu, 2013; Yan \& Özbay, 2008). In Çalıșkan et al. (2013), the calculation of dwell time is formulated as a semi-definite programming (SDP) in terms of linear matrix inequalities (LMIs). Piecewise Lyapunov-Krasovskii functionals are derived by model transformation methods. The upper bound of the derivative of the Lyapunov function is minimised which ends up with a sub-optimal solution to the dwell time minimisation problem. In Yan, Özbay, and Sansal (2011), parameter-varying systems with time-delays are stabilised by switching control. The resulting dwell time is minimised with iterative search methods. The present paper proposes a quasi-convex optimisation approach to directly minimise the dwell time and converges to global minimum of represented upper bound of the dwell time. To reduce conservatism due to model transformations, we derive the stability conditions by using free-weighting matrices.

The notation to be used in the paper is standard: $\mathbb{R}$ $\left(\mathbb{R}^{+}, \mathbb{R}_{0}^{+}\right)$stands for the set of real numbers (positive real numbers, non-negative real numbers), $\mathcal{C}$ is used to denote the set of differentiable functions, $\mathbb{Z}^{+}$symbolises the set of positive integers. The identity matrices are denoted by $I$. We use $X \succ 0(\succeq, \prec, \preceq 0)$ to denote a positive definite (positive-semidefinite, negative definite, negativesemidefinite) matrix. $\sigma_{\max }[X]$ and $\sigma_{\min }[X]$ denote the maximum and minimum singular values of $X$, respectively. The asterisk symbol $(*)$ denotes complex conjugate transpose of a matrix and $x_{t}$ denotes the translation operator acting on the trajectory such as $x_{t}(\theta)=$ $x(t+\theta)$ for some non-zero interval $\theta \in[-\tau, 0]$. The operator $\operatorname{diag}\left[X_{1}, X_{2}, \ldots, X_{n}\right]$ denotes a block diagonal matrix whose elements on the main block diagonal are $X_{1}, X_{2}, \ldots, X_{n}$. The norm $\|\cdot\|$ is defined as the Eucledian norm for a vector in $\mathbb{R}^{n}$ and the norm on $\mathcal{C}$ is defined as follows:

$$
|f|_{[a, b]}=\max \left\{\sup _{t \in[a, b]}\|f(t)\|, \sup _{t \in[a, b]}\|\dot{f}(t)\|\right\} .
$$

Rest of the paper is organised as follows. In Section 2, preliminaries and problem definition are introduced. In Section 3, dwell time stability condition is given. In Section 4, quasi-convex optimisation of the upper bound of the dwell time and some numerical examples are proposed. In Section 5, dwell time minimising controller synthesis is presented with some numerical examples to illustrate effectiveness of the proposed algorithm. Conclusions are summarised in Section 6.

\section{Preliminaries and problem definition}

Consider a class of switched delay system given by

$\Sigma_{\sigma(t)}:\left\{\begin{aligned} \dot{x}(t) & =A_{\sigma(t)} x(t) & & \\ & +\bar{A}_{\sigma(t)} x\left(t-r_{\sigma(t)}(t)\right), & & t \geq 0 \\ x(\theta) & =\varphi(\theta), & & \forall \theta \in\left[-\tau_{\max }, 0\right]\end{aligned}\right.$

where $x(t) \in \mathbb{R}^{n}$ is the pseudo-state and $\sigma(t)$ is the piecewise switching signal such that $\sigma(t): \mathbb{R}^{+} \rightarrow \mathcal{P}, \mathcal{P}:=$ $\{1,2, \ldots, m\}$ is an index set, $m \in \mathbb{Z}^{+}$is the number of subsystems and initial condition belongs to Banach space of continuous functions such that $\varphi(\cdot) \in \mathcal{C}$. Time-delay, $r_{\sigma(t)}(t)$, is a time-varying differentiable function that satisfies

$$
\begin{gathered}
0 \leq r_{\sigma(t)}(t) \leq \tau_{\sigma(t)}, \\
\left|\dot{r}_{\sigma(t)}(t)\right| \leq d_{\sigma(t)},
\end{gathered}
$$

where $\tau_{\sigma(t)}, d_{\sigma(t)}>0$ are piecewise constants. We introduce the quadropule

$$
\Sigma_{i}:=\left(A_{i}, \bar{A}_{i}, \tau_{i}, d_{i}\right) \in \mathbb{R}^{n \times n} \times \mathbb{R}^{n \times n} \times \mathbb{R} \times \mathbb{R}
$$

to describe the $i$ th candidate subsystem of Equation (1) and $\tau_{\max }=\max _{i \in \mathcal{P}} \tau_{i}$.

Definition 2.1: A switched delay system is stable if there exists a function $\beta$ of class $\mathcal{K}$ such that

$$
\|x(t)\| \leq \beta\left(|x|_{\left[t_{0}-\tau_{\max }, t_{0}\right]}\right)
$$


along every solution of Equation (1). Furthermore, a switched delay system is asymptotically stable when it is stable and $\lim _{t \rightarrow+\infty} x(t)=0$.

Lemma 2.1: (See Gu et al., 2003). Consider the nonswitched linear subsystem $\Sigma_{i}$ of the system (1) for an $i \in$ $\mathcal{P}$. Suppose $u_{i}, v_{i}, w_{i}: \mathbb{R}_{0}^{+} \rightarrow \mathbb{R}_{0}^{+}$are continuous, nondecreasing functions satisfying $u_{i}(0)=v_{i}(0)=0, w_{i}(s)>$ 0 for $s>0$. If there exists a continuous functional $V$, such that

$$
\begin{aligned}
& u_{i}(\|x(t)\|) \leq V_{i}\left(t, x_{t}\right) \leq v_{i}\left(|x|_{\left[t-\tau_{i}, t\right]}\right), \quad \forall t \geq t_{0} \\
& \dot{V}_{i}\left(t, x_{t}\right) \leq-w_{i}(\|x(t)\|), \quad \forall t \geq t_{0}
\end{aligned}
$$

then the solution $x=0$ of the subsystem $\Sigma_{i}$ is uniformly asymptotically stable.

Let us construct the following piecewise Lyapunov function:

$$
\begin{aligned}
V_{i}\left(t, x_{t}\right):= & x^{T}(t) P_{i} x(t)+\int_{t-r_{i}(t)}^{t} x^{T}(s) Q_{i} x(s) d s \\
& +\int_{-\tau_{i}}^{0} \int_{t+\theta}^{t} \dot{x}^{T}(s) Z_{i} \dot{x}(s) d s d \theta, \quad \forall i \in \mathcal{P}
\end{aligned}
$$

Lemma 2.2: (See Wu, He, \& She, 2010). Consider the nonswitched linear subsystem $\Sigma_{i}$ for an $i \in \mathcal{P}$ of the switched system (1) with varying delays, $r_{i}(t)$. Given scalar $\tau_{i}>0$ and $d_{i}>0$ for which both Equations (2) and (3) hold, the $i$ th subsystem is asymptotically stable if there exist symmetric matrices $P_{i} \succ 0, Q_{i} \succeq 0, Z_{i} \succ 0$, and

$$
X_{i}:=\left[\begin{array}{cc}
X_{11 i} & X_{12 i} \\
* & X_{22 i}
\end{array}\right] \succeq 0,
$$

and any appropriately dimensioned matrices $N_{1 i}$ and $N_{2 i}$ such that the following LMIs hold:

$$
\begin{aligned}
\phi_{i} & :=\left[\begin{array}{ccc}
\phi_{11 i} & \phi_{12 i} & \tau_{i} A_{i}^{T} Z_{i} \\
* & \phi_{22 i} & \tau_{i} \bar{A}_{i}^{T} Z_{i} \\
* & * & -\tau_{i} Z_{i}
\end{array}\right] \prec 0, \\
\psi_{i} & :=\left[\begin{array}{ccc}
X_{11 i} & X_{12 i} & N_{1 i} \\
* & X_{22 i} & N_{2 i} \\
* & * & Z_{i}
\end{array}\right] \succeq 0,
\end{aligned}
$$

where

$$
\begin{aligned}
& \phi_{11 i}=P_{i} A_{i}+A_{i}^{T} P_{i}+N_{1 i}+N_{1 i}^{T}+Q_{i}+\tau_{i} X_{11 i}, \\
& \phi_{12 i}=P_{i} \bar{A}_{i}-N_{1 i}+N_{2 i}^{T}+\tau_{i} X_{12 i}, \\
& \phi_{22 i}=-N_{2 i}-N_{2 i}^{T}-\left(1-d_{i}\right) Q_{i}+\tau_{i} X_{22 i} .
\end{aligned}
$$

\section{Main results}

The following proposition is a modified version of a result obtained in Çalışkan et al. (2013). In the corresponding proposition, the time $T_{*}$ is calculated as the time instant after which norm of the states does not exceed the predefined parameter $\rho$ for the non-switched case. Furthermore, after the dwell time $T_{*}+\tau_{\max }$ the norm of the state functional does not exceed $\rho$. Note that the norm of the

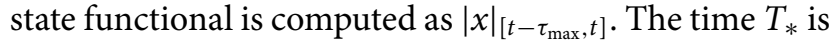
related to the upper bounds defined in Lemma 2.1.

Proposition 3.1: For any non-switching linear subsystem $\Sigma_{i}$ satisfying Lemma 2.1 with $\lim _{s \rightarrow \infty} u_{i}(s) \rightarrow \infty$, assume there exists a non-decreasing function $u_{d_{i}}$ such that

$$
u_{d_{i}}(\|\dot{x}(t)\|) \leq V_{i}\left(t, x_{t}\right) .
$$

For an arbitrary $\rho, 0<\rho<\delta_{2},|x|_{\left[t_{0}-\tau_{i}, t_{0}\right]} \leq \delta_{1}$ implies

$$
|x|_{\left[t-\tau_{\max }, t\right]} \leq \rho, \quad \forall t>t_{0}+\tau_{\max }+T_{i}\left(\delta_{1}, \rho\right)
$$

where $v_{i}$ and $w_{i}$ are defined as in Lemma 2.1, $u\left(\delta_{2}\right)=v\left(\delta_{1}\right)$ and

$$
T_{i}\left(\delta_{1}, \rho\right)=\frac{v_{i}\left(\delta_{1}\right)}{w_{i}(\rho)} .
$$

Proof: Let $T_{*}>0$ and let $\left\|x\left(t_{1}\right)\right\|>\rho$ for a time instant $t_{1}>t_{0}+T_{*}$. Function $w_{i}$ is non-decreasing by definition, as a result inf $\rho_{\rho<s<\delta_{2}} w_{i}(s)=w_{i}(\rho)$. Since the subsystem $\Sigma_{i}$ is stable and $V_{i}$ is a Lyapunov-Krasovskii functional, from Lemma 2.1, we have the following:

$$
\dot{V}_{i}\left(t, x_{t}\right) \leq-w_{i}(\rho), \quad t_{0} \leq t \leq t_{1} .
$$

This implies

$$
\begin{aligned}
V_{i}\left(t, x_{t}\right) & \leq V_{i}\left(t_{0}, x_{0}\right)-\left(t-t_{0}\right) w_{i}(\rho) \\
& \leq v_{i}\left(\delta_{1}\right)-\left(t-t_{0}\right) w_{i}(\rho) .
\end{aligned}
$$

Let $T_{*}>v_{i}\left(\delta_{1}\right) / w_{i}(\rho)$. Then for every $t>t_{0}+T_{*}$, we have $V_{i}\left(t, x_{t}\right) \leq 0$. However, we assume that there is a time instant $t_{1}>t_{0}+T_{*}$ such that $\left\|x\left(t_{1}\right)\right\|>\rho$. This implies that

$$
V_{i}\left(t_{1}, x_{t_{1}}\right) \geq u_{i}\left(\left\|x\left(t_{1}\right)\right\|\right)>u_{i}(\rho)>0
$$

This is a contradiction. Therefore, time instant $t_{1}$ cannot exist and this implies

$$
\|x(t)\| \leq \rho, \quad \forall t>t_{0}+\frac{v_{i}\left(\delta_{1}\right)}{w_{i}(\rho)} .
$$


Similarly, assuming there is a time instant $t_{1}>t_{0}+T_{*}$ such that $\left\|\dot{x}\left(t_{1}\right)\right\| \geq \rho$

$$
V_{i}\left(t_{1}, x_{t_{1}}\right) \geq u_{d_{i}}\left(\left\|\dot{x}\left(t_{1}\right)\right\|\right)>u_{d_{i}}(\rho)>0
$$

which is also a contradiction. Hence,

$$
\|x(t)\|<\rho, \quad\|\dot{x}(t)\|<\rho, \quad \forall t>t_{0}+T_{*}
$$

If we wait for a period of maximum time-delay such that $t>t_{0}+T_{*}+\tau_{\max }$, the inequality $|x(t)|_{\left[t-\tau_{\max }, t\right]} \leq \rho$ holds, which concludes the proof.

Now, some specific upper and lower bounds for the Lyapunov function (6) can be given as $v_{i}(s):=\mu_{i} s^{2}$ with

$$
\mu_{i}:=\sigma_{\max }\left[P_{i}\right]+\tau_{i}^{2} \sigma_{\max }\left[Q_{i}\right]+\frac{1}{2} \tau_{i}^{2} \sigma_{\max }\left[Z_{i}\right]
$$

and

$$
u_{i}(s)=\sigma_{\min }\left[P_{i}\right] s^{2},
$$

respectively. Another lower bound of the Lyapunov function with respect to norm of $\dot{x}(t)$ can be defined as

$$
u_{d_{i}}(s):=\frac{1}{2} \tau_{i}^{2} \sigma_{\min }\left[Z_{i}\right] s^{2}
$$

where $u_{d_{i}}(\|\dot{x}(t)\|) \leq V_{i}\left(t, x_{t}\right)$.

The upper bounds $v_{i}(s)$ can be calculated via LMI conditions defined in Lemma 2.2 due to Equation (10). In order to formulate the upper bounds of the derivative of the Lyapunov functions $w_{i}(s)$ as an LMI feasibility problem, we need a new result, stated as Proposition 3.2.

Remark 3.1: In the proof of Proposition 3.2, an inequality from the proof of Lemma 2.2 in Wu et al. (2010) will be used, specifically:

$$
\dot{V}_{i}\left(t, x_{t}\right) \leq \eta_{1}^{T}(t) \Xi_{i} \eta_{1}(t)-\int_{t-r_{i}(t)}^{t} \eta_{2}^{T}(t, s) \psi_{i} \eta_{2}(t, s) d s
$$

where $\psi_{i}$ is defined in Equation (9) and

$$
\begin{aligned}
\eta_{2}(t, s) & =\left[x^{T}(t), x^{T}\left(t-r_{i}(t)\right), \dot{x}^{T}(s)\right]^{T} \\
\Xi_{i} & =\left[\begin{array}{cc}
\phi_{11 i}+\tau_{i} A_{i}^{T} Z_{i} A_{i} & \phi_{12 i}+\tau_{i} A_{i}^{T} Z_{i} \bar{A}_{i} \\
* & \phi_{22 i}+\tau_{i} \bar{A}_{i}^{T} Z_{i} \bar{A}_{i}
\end{array}\right] .
\end{aligned}
$$

Note that Equation (8) is the Schur complement of $\Xi_{i}$. For more information about the proof, we refer to $\mathrm{Wu}$ et al. (2010).

Proposition 3.2: Consider the system (1) with each $\Sigma_{i}$ satisfying Lemma 2.2, if there exist matrices $W_{i}^{T}=W_{i} \succeq 0$ such that following LMIs hold:

$$
\bar{\phi}_{i}:=\left[\begin{array}{ccc}
\phi_{11 i}+W_{i} & \phi_{12 i} & \tau_{i} A_{i}^{T} Z_{i} \\
& \phi_{22 i} & \tau_{i} \bar{A}_{i}^{T} Z_{i} \\
& * & -\tau_{i} Z_{i}
\end{array}\right] \prec 0, \quad \forall i \in \mathcal{P}
$$

then $\dot{V}_{i}\left(t, x_{t}\right) \leq-x^{T}(t) W_{i} x(t)$ for all $i \in \mathcal{P}$.

Proof: Consider the inequality (12). Since $\psi_{i} \succeq 0$, we know that $\dot{V}_{i}\left(t, x_{t}\right) \leq \eta_{1}^{T}(t) \Xi_{i} \eta_{1}(t)$. Bounding this inequality,

$$
\eta_{1}^{T}(t) \Xi_{i} \eta_{1}(t) \leq-x^{T}(t) W_{i} x(t)
$$

yields $\eta_{1}^{T}(t) D_{i} \eta_{1}(t) \leq 0$ where

$$
D_{i}:=\left[\begin{array}{ll}
\phi_{11 i}+W_{i}+\tau_{i} A_{i}^{T} Z_{i} A_{i} & \phi_{12 i}+\tau_{i} A_{i}^{T} Z_{i} \bar{A}_{i} \\
& \phi_{22 i}+\tau_{i} \bar{A}_{i}^{T} Z_{i} \bar{A}_{i}
\end{array}\right] .
$$

Since $\bar{\phi}_{i}$ is the Schur complement of $D_{i}$, if Equation (13) holds, then $\dot{V}_{i}\left(t, x_{t}\right) \leq-x^{T}(t) W_{i} x(t)$.

After defining a new variable

$$
\lambda_{i}:=\sigma_{\min }\left[W_{i}\right],
$$

we can select the upper bound function for the derivative of the Lyapunov function as $w_{i}(s)=\lambda_{i} s^{2}$.

Assume that the Lemma 2.1 is satisfied for the system (1). There exists a $\delta_{2}>\delta_{1}>0$ such that $u\left(\delta_{2}\right)=v\left(\delta_{1}\right)$. For such $\delta_{2}$, Lemma 2.1 implies that $\|x(t)\| \leq \delta_{2}$ for all $t>t_{0}$ if $|x|_{\left[t_{0}-\tau_{i}, t_{0}\right]} \leq \delta_{1}$. Hence, for $u(s)$ and $v(s)$ defined in Equations (10) and (11), following inequality holds:

$$
\|x(t)\| \leq \beta|x|_{\left[t_{0}-\tau_{i}, t_{0}\right]}, \quad \forall i \in \mathcal{P}
$$

where

$$
\beta=\max _{i \in \mathcal{P}} \sqrt{\frac{\mu_{i}}{\sigma_{\min }\left[P_{i}\right]}} .
$$

Consider the $k$ th switching instant $t_{k}$. The dwell time $\tau_{D}$ is defined as the time instant after which the norm of the state functionals for any $t_{k}>t_{k-1}+\tau_{D}$ does not exceed the norm of the state functional at time $t_{k-1}$. Hence, $\rho$ in Proposition 3.1 is defined as a fraction of the norm of the state functional at the switching instant $t_{k-1}$. As a result, the dwell time defined in this paper should be strictly greater than the maximum of all the possible delays. The fraction is a pre-defined number $\alpha \in(0,1)$.

Theorem 3.1: Consider the switched delay system described in Equation (1). Assume all of the candidate subsystems satisfy Lemma 2.2. Then, the switched delay system is asymptotically stable for all switching signals 
satisfying dwell time requirement $\tau_{D}$

$$
\tau_{D}=\frac{1}{\alpha^{2}} \max _{i \in \mathcal{P}} \frac{\mu_{i}}{\lambda_{i}}+\tau_{\max }, \quad \text { for any } \alpha \in(0,1)
$$

with $\mu_{i}$ and $\lambda_{i}$ being defined in Equations (10) and (14), respectively.

Proof: Let us choose $\rho=\alpha \delta_{k-1}$ where $\delta_{k}$ denotes norm of the state at the $k$ th switching instant such that $\delta_{k}=$ $|x|_{\left[t_{k}-\tau_{\max }, t_{k}\right]}$. Let us restrict ourselves to switching signals to signals for which the time interval between two consecutive switching instants is larger than dwell time such that $t_{k}-t_{k-1}>\tau_{D}$. Introducing this dwell time as

$$
\tau_{D}=\max _{i \in \mathcal{P}} T_{i}\left(\delta_{k-1}, \alpha \delta_{k-1}\right)+\tau_{\max }
$$

leads us to an inequality from Proposition 3.1 as

$$
|x|_{\left[t_{k}-\tau_{\max }, t_{k}\right]} \leq \alpha|x|_{\left[t_{k-1}-\tau_{\max }, t_{k-1}\right]}, \quad \forall t_{k}>t_{k-1}+\tau_{D}
$$

where

$$
T_{i}\left(\delta_{k-1}, \alpha \delta_{k-1}\right)=\frac{v_{i}\left(\delta_{k-1}\right)}{w_{i}\left(\alpha \delta_{k-1}\right)}=\frac{\mu_{i}}{\alpha^{2} \lambda_{i}}
$$

From Equations (15) and (17),

$$
\begin{aligned}
\|x(t)\| \leq & \beta|x|_{\left[t_{k}-\tau_{\max }, t_{k}\right]} \\
\leq & \beta \alpha|x|_{\left[t_{k-1}-\tau_{\max }, t_{k-1}\right]} \\
& \vdots \\
\leq & \beta \alpha^{k}|x|_{\left[t_{0}-\tau_{\max }, t_{0}\right]}, \quad \forall \alpha \in(0,1), \\
\leq & \beta \alpha|x|_{\left[t_{0}-\tau_{\max }, t_{0}\right]}, \quad \forall \alpha \in(1)
\end{aligned}
$$

which is satisfying the stability condition described in Definition 2.1.

Remark 3.2: The parameter $\alpha$ is the ratio of the norms of the state functionals at the consecutive switching instants as in Equation (17). Hence, it can be regarded as a measure of the decay rate. This parameter quantifies a tradeoff between the dwell time and the decay rate, i.e. the larger $\alpha$, the smaller dwell time but the slower decay rate (it should be strictly less than 1 for stability of the switched system).

\section{Minimum dwell time via quasi-convex optimisation}

In order to minimise the dwell time in Equation (16), we can define the optimisation problem with a cost function $f\left(\mu_{i}, \lambda_{i}\right):=\max _{i \in \mathcal{P}} \mu_{i} / \lambda_{i}$ for a given $\alpha$. This is a quasiconvex function since it is the composition of a convex function with a nondecreasing function (Bullo \& Liberzon, 2006). It is known that an optimisation problem with a quasi-convex cost function and convex constraints can be solved by iterative methods such as bisection algorithm (Boyd \& Vandenberghe, 2004).

We define a new free variable $t$ to bound the cost function:

$$
\frac{\mu_{i}}{\lambda_{i}} \leq t, \quad \forall i \in \mathcal{P}
$$

The parameters $\mu_{i}$ and $\lambda_{i}$ are related with the eigenvalues of $P_{i}, Q_{i}, Z_{i}$ and $W_{i}$ as in Equations (10) and (14). We define $p_{i}, q_{i}$ and $z_{i}$ to define maximum eigenvalues of $P_{i}$, $Q_{i}$ and $Z_{i}$, respectively. So, the inequality (18) can be rewritten as $p_{i}+\tau_{i} q_{i}+\frac{1}{2} \tau_{i}^{2} z_{i}-t \lambda_{i}<0$.

With respect to the free parameters $P_{i}, Q_{i}, Z_{i}, W_{i}, X_{11 i}$, $X_{12 i}, X_{22 i}, N_{1 i}, N_{2 i} p_{i}, q_{i}, z_{i}, w_{i}, t_{u}$, the upper bound of the dwell time is minimised via following optimisation problem:

$$
\begin{array}{lll}
\text { minimise } & t \\
\text { subject to } & \operatorname{diag}\left[P_{i}, Q_{i}, Z_{i}, W_{i}, X_{i}\right] \succ 0, \quad \forall i \in \mathcal{P} \\
& \operatorname{diag}\left[P_{i}, Q_{i}, Z_{i},-W_{i}\right] & \\
& \prec \operatorname{diag}\left[p_{i} I, q_{i} I, z_{i} I,-\lambda_{i} I\right], \quad \forall i \in \mathcal{P} \\
& \psi_{i} \succeq 0, \bar{\phi}_{i} \prec 0, \quad \forall i \in \mathcal{P} & \\
& p_{i}+\tau_{i} q_{i}+\frac{1}{2} \tau_{i}^{2} z_{i}-t \lambda_{i}<0, \quad \forall i \in \mathcal{P}
\end{array}
$$

where $X_{i}, \psi_{i}$ and $\bar{\phi}_{i}$ are defined in Equations (7), (9) and (13), respectively. Then, the dwell time can be chosen as $\tau_{D}=\alpha t+\tau_{\max }$ for any $\alpha \in(0,1)$. However, the optimisation problem involves a bilinear matrix inequality when $t$ is considered as a free parameter.

Searching for minimum $t$ with bisection algorithm generates a sequence of linear SDP feasibility problems which can easily be solved by SeDuMi Sturm (1999).

In this section, the examples are taken from published papers for comparison purposes. Examples 4.1-4.3 can be found in Çalışkan et al. (2013), Yan and Özbay (2008) and Chen and Zheng (2010), respectively.

Example 4.1: Let $\Sigma_{1}$ and $\Sigma_{2}$ be

$$
\begin{aligned}
A_{1}=\left[\begin{array}{cc}
-2 & 0 \\
0 & -0.9
\end{array}\right], & \bar{A}_{1}=\left[\begin{array}{cc}
-1 & 0 \\
-0.5 & -1
\end{array}\right], \\
\tau_{1}=0.3 s, & d_{1}=0 \\
A_{2}=\left[\begin{array}{cc}
-1 & 0.5 \\
0 & -1
\end{array}\right], & \bar{A}_{2}=\left[\begin{array}{cc}
-1 & 0 \\
0.1 & -1
\end{array}\right], \\
\tau_{2}=0.6 s, & d_{2}=0 .
\end{aligned}
$$




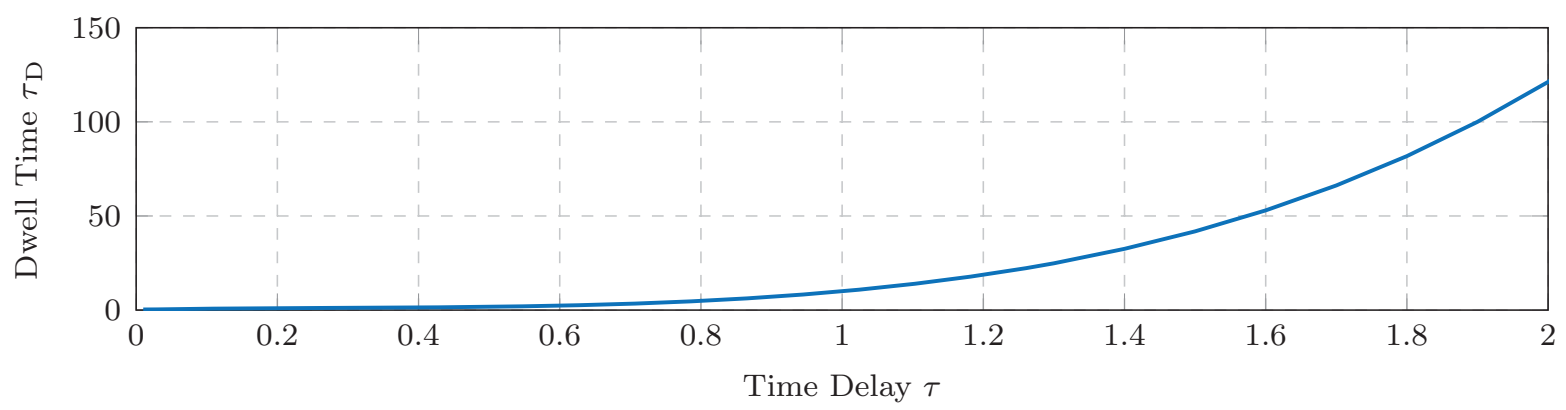

Figure 1. Dwell time results for different delay values of the switched delay system described in the Example 4.1 where delays are fixed such that $d_{1}=d_{2}=0$ with upper bounds $\tau_{1}=\tau$ and $\tau_{2}=2 \tau$.

Table 1. Dwell time for different $\tau_{i}$ and $d_{i}$ values of Example $4.1, \alpha=0.99$.

\begin{tabular}{lcccc}
\hline$\tau_{1}$ & $\tau_{2}$ & $d_{1}$ & $d_{2}$ & $\tau_{D}$ \\
\hline $0.15 \mathrm{~s}$ & $0.3 \mathrm{~s}$ & $0 \mathrm{~s}$ & $0 \mathrm{~s}$ & $0.69 \mathrm{~s}$ \\
$0.15 \mathrm{~s}$ & $0.3 \mathrm{~s}$ & $0.15 \mathrm{~s}$ & $0.3 \mathrm{~s}$ & $0.69 \mathrm{~s}$ \\
$0.3 \mathrm{~s}$ & $0.6 \mathrm{~s}$ & $0 \mathrm{~s}$ & $0 \mathrm{~s}$ & $1.11 \mathrm{~s}$ \\
$0.3 \mathrm{~s}$ & $0.6 \mathrm{~s}$ & $0.3 \mathrm{~s}$ & $0.3 \mathrm{~s}$ & $1.11 \mathrm{~s}$ \\
$0.3 \mathrm{~s}$ & $0.6 \mathrm{~s}$ & $0.6 \mathrm{~s}$ & $0.6 \mathrm{~s}$ & $1.11 \mathrm{~s}$ \\
$0.6 \mathrm{~s}$ & $1.2 \mathrm{~s}$ & $0 \mathrm{~s}$ & $0 \mathrm{~s}$ & $2.54 \mathrm{~s}$ \\
$0.6 \mathrm{~s}$ & $1.2 \mathrm{~s}$ & $0.3 \mathrm{~s}$ & $0.3 \mathrm{~s}$ & $2.76 \mathrm{~s}$ \\
$0.6 \mathrm{~s}$ & $1.2 \mathrm{~s}$ & $0.6 \mathrm{~s}$ & $0.6 \mathrm{~s}$ & $3.51 \mathrm{~s}$ \\
\hline
\end{tabular}

Table 2. Dwell time for different $\tau_{i}$ and $d_{i}$ values of Example $4.2, \alpha=0.99$.

\begin{tabular}{lcccc}
\hline$\tau_{1}$ & $\tau_{2}$ & $d_{1}$ & $d_{2}$ & $\tau_{D}$ \\
\hline $0.08 \mathrm{~s}$ & $0.1 \mathrm{~s}$ & $0 \mathrm{~s}$ & $0 \mathrm{~s}$ & $0.46 \mathrm{~s}$ \\
$0.16 \mathrm{~s}$ & $0.2 \mathrm{~s}$ & $0.15 \mathrm{~s}$ & $0.15 \mathrm{~s}$ & $0.58 \mathrm{~s}$ \\
$0.3 \mathrm{~s}$ & $0.4 \mathrm{~s}$ & $0.2 \mathrm{~s}$ & $0.2 \mathrm{~s}$ & $0.84 \mathrm{~s}$ \\
$0.6 \mathrm{~s}$ & $0.8 \mathrm{~s}$ & $0 \mathrm{~s}$ & $0 \mathrm{~s}$ & $1.38 \mathrm{~s}$ \\
$0.9 \mathrm{~s}$ & $1.2 \mathrm{~s}$ & $0 \mathrm{~s}$ & $0 \mathrm{~s}$ & $2.39 \mathrm{~s}$ \\
$0.9 \mathrm{~s}$ & $1.2 \mathrm{~s}$ & $0.3 \mathrm{~s}$ & $0.3 \mathrm{~s}$ & $2.58 \mathrm{~s}$ \\
$0.9 \mathrm{~s}$ & $1.2 \mathrm{~s}$ & $0.6 \mathrm{~s}$ & $0.6 \mathrm{~s}$ & $3.15 \mathrm{~s}$ \\
$0.9 \mathrm{~s}$ & $1.2 \mathrm{~s}$ & $0.9 \mathrm{~s}$ & $0.9 \mathrm{~s}$ & $176.70 \mathrm{~s}$ \\
\hline
\end{tabular}

Dwell time results for different delay values for this example can be seen in Figure 1. Corresponding minimum dwell times for different $\tau_{i}$ and $d_{i}$ values are illustrated in Table 1.

Example 4.2: Let $\Sigma_{1}$ and $\Sigma_{2}$ be

$$
\begin{aligned}
& A_{1}=\left[\begin{array}{cc}
-1.799 & -0.814 \\
0.2 & -0.714
\end{array}\right], \quad \bar{A}_{1}=\left[\begin{array}{cc}
-1 & 0 \\
-0.45 & -1
\end{array}\right], \\
& \tau_{1}=0.155 s, \quad d_{1}=0 . \\
& A_{2}=\left[\begin{array}{cc}
-1.853 & -0.093 \\
-0.853 & -1.1593
\end{array}\right], \quad \bar{A}_{2}=\left[\begin{array}{cc}
-1 & 0 \\
0.05 & -1
\end{array}\right] \text {, } \\
& \tau_{2}=0.2 s, \quad d_{2}=0 .
\end{aligned}
$$

Corresponding minimum dwell times for different $\tau_{i}$ and $d_{i}$ values are illustrated in Table 2 . Note that second subsystem of Example 4.2 is unstable for $d_{2}>0.905$. As $d_{2}$
Table 3. Dwell time for $\alpha=0.99$.

\begin{tabular}{lccc}
\hline Example & 1 & 2 & 3 \\
\hline Chen and Zheng (2010) & - & - & $43.99 \mathrm{~s}$ \\
Yan and Özbay (2008) & $6.51 \mathrm{~s}$ & - & - \\
Çalıskan et al. (2013) & $3.4 \mathrm{~s}$ & $0.72 \mathrm{~s}$ & - \\
Present work & $1.11 \mathrm{~s}$ & $0.58 \mathrm{~s}$ & $16.60 \mathrm{~s}$ \\
\hline
\end{tabular}

coming closer to the stability limits, dwell time increases dramatically, that sits in shaded row of Table 2 .

Example 4.3: This example is the Case 3 of Example 4.1 from the paper (Chen \& Zheng, 2010). Let $\Sigma_{1}$ be

$$
\begin{array}{rr}
A_{1}=\left[\begin{array}{cc}
0 & 1 \\
-10 & -1
\end{array}\right], & \bar{A}_{1}=0.9 \cdot\left[\begin{array}{cc}
0.1 & 0 \\
-0.01 & 0.05
\end{array}\right], \\
\tau_{1}=1.82, & d_{1}=0, \\
A_{2}=\left[\begin{array}{cc}
0 & 1 \\
-0.1 & -0.5
\end{array}\right], & \bar{A}_{2}=0.7 \cdot\left[\begin{array}{cc}
0.02 & 0 \\
-0.01 & 0.02
\end{array}\right], \\
\tau_{2}=1.82, & d_{2}=0,
\end{array}
$$

Comparison of present paper with previous works for Examples 4.1-4.3 can be seen in Table 3.

Example 1.1: This example is a slighlty modified version of Example 4.1 of Sun and Ge (2011) with $a=50$, where $a$ is a parameter used in corresponding example. In the example, system is not guaranteed stable under arbitrary switching.

Let the subsystems be:

$$
\begin{array}{rr}
A_{1}=\left[\begin{array}{ll}
-0.1 & 1.1 \\
-0.9 & -1
\end{array}\right], & \bar{A}_{1}=\left[\begin{array}{cc}
0.05 & -0.1 \\
-0.1 & 0
\end{array}\right], \\
\tau_{1}=0.01, & d_{1}=0.2 \\
A_{2}=\left[\begin{array}{cc}
-0.1 & 1 \\
-150 & -50
\end{array}\right], & \bar{A}_{2}=\left[\begin{array}{cc}
0.05 & 0 \\
-1 & -1
\end{array}\right], \\
\tau_{2}=0.05, & d_{2}=0.1 .
\end{array}
$$

Resulting dwell time is $\tau_{D}=1.698$ with $T_{*}=1.648$. 


\section{Dwell time minimising controller synthesis}

Consider a class of switched delay systems given by

$\tilde{\Sigma}_{\sigma(t)}:\left\{\begin{aligned} \dot{x}(t)= & A_{\sigma(t)} x(t) & & \\ & +\bar{A}_{\sigma(t)} x\left(t-r_{\sigma(t)}(t)\right) & & \\ & +B_{\sigma(t)} u(t), & & t \geq 0 \\ x(\theta)= & \varphi(\theta), & & \forall \theta \in\left[-\tau_{\max }, 0\right]\end{aligned}\right.$

We introduce the quintet

$\tilde{\Sigma}_{i}:=\left(A_{i}, \bar{A}_{i}, B_{i}, \tau_{i}, d_{i}\right) \in \mathbb{R}^{n \times n} \times \mathbb{R}^{n \times n} \times \mathbb{R}^{n \times m} \times \mathbb{R} \times \mathbb{R}$

to describe the ith candidate subsystem of Equation (20) and $\tau_{\max }=\max _{i \in \mathcal{P}} \tau_{i}$.

Lemma 5.1: (See Wu et al., 2010). Consider any nonswitching linear subsystem $\tilde{\Sigma}_{i}$ of the switched delay system (20) with a delay, $r_{i}(t)$. For given scalar $\tau_{i}$ and $d_{i}$ which both Equations (2) and (3) hold, if there exist matrices $L_{i}>$ $0, T_{i} \geq 0, R_{i}>0$, and

$$
Y_{i}:=\left[\begin{array}{cc}
Y_{11 i} & Y_{12 i} \\
* & Y_{22 i}
\end{array}\right] \succeq 0
$$

and any appropriately dimensioned matrices $M_{1 i}, M_{2 i}$ and $V_{i}$ such that the following matrix inequalities hold:

$$
\begin{aligned}
\Pi_{i} & =\left[\begin{array}{ccc}
\Pi_{11 i} & \Pi_{12 i} & \tau_{i}\left(L_{i} A_{i}^{T}+V_{i}^{T} B_{i}^{T}\right) \\
* & \Pi_{22 i} & \tau_{i} L_{i} \bar{A}_{i}^{T} \\
* & * & -\tau_{i} R_{i}
\end{array}\right] \prec 0, \\
\Lambda_{i} & =\left[\begin{array}{ccc}
Y_{11 i} & Y_{12 i} & M_{1 i} \\
* & Y_{22 i} & M_{2 i} \\
* & * & L_{i} R_{i}^{-1} L_{i}
\end{array}\right] \succeq 0,
\end{aligned}
$$

where

$$
\begin{aligned}
\Pi_{11 i}= & L_{i} A_{i}^{T}+A_{i} L_{i}+B_{i} V_{i}+V_{i}^{T} B_{i}^{T}+M_{1 i} \\
& +M_{1 i}^{T}+T_{i}+\tau_{i} Y_{11 i} \\
\Pi_{12 i}= & \bar{A}_{i} L_{i}-M_{1 i}+M_{2 i}^{T}+\tau_{i} Y_{12 i} \\
\Pi_{22 i}= & -M_{2}-M_{2}^{T}-\left(1-d_{i}\right) T_{i}+\tau_{i} Y_{22 i}
\end{aligned}
$$

then the subsystem $\Sigma_{i}$ can be stabilised by control law $u(t)=K_{i} x(t)$, and the controller gain is $K_{i}=V_{i} L_{i}^{-1}$.

Proof: After applying memoryless state-feedback controller to closed-loop system

$$
\dot{x}(t)=\left(A_{i}+B_{i} K_{i}\right) x(t)+\bar{A}_{i} x\left(t-r_{i}(t)\right),
$$

let us replace the $A_{i}$ with $A_{i}+B_{i} K_{i}$, pre- and postmultiply (8) by $\operatorname{diag}\left[P_{i}^{-1}, P_{i}^{-1}, Z_{i}^{-1}\right]$, pre- and postmultiply (9) by $\operatorname{diag}\left[P_{i}^{-1}, P_{i}^{-1}, P_{i}^{-1}\right]$, and make the following change of variables:

$$
\begin{aligned}
L_{i} & :=P_{i}^{-1}, \quad T_{i}:=P_{i}^{-1} Q_{i} P_{i}^{-1}, \quad R_{i}:=Z_{i}^{-1} \\
M_{1 i} & :=P_{i}^{-1} N_{1 i} P_{i}^{-1}, \quad M_{2 i}:=P_{i}^{-1} N_{2 i} P_{i}^{-1}, \quad V_{i}=K_{i} P_{i}^{-1} \\
Y_{i} & :=\operatorname{diag}\left[P_{i}^{-1}, P_{i}^{-1}\right] \cdot X_{i} \cdot \operatorname{diag}\left[P_{i}^{-1}, P_{i}^{-1}\right]
\end{aligned}
$$

These operations end up with Equations (21) and (22) which complete the proof.

Due to the term $L_{i} R_{i}^{-1} L_{i}$, condition (22) in Lemma 5.1 is not an LMI. In order to handle this term, let us define a new variable, $S_{i}$, for which

$$
L_{i} R_{i}^{-1} L_{i} \succeq S_{i},
$$

and replace Equation (22) with

$$
\bar{\Lambda}_{i}:=\left[\begin{array}{ccc}
Y_{11 i} & Y_{12 i} & M_{1 i} \\
* & Y_{22} & M_{2 i} \\
* & * & S_{i}
\end{array}\right] \succeq 0 .
$$

Inequality (24) is equivalent to $L^{-1} R L^{-1} \preceq S^{-1}$, which the Schur complement allows us to write as

$$
\left[\begin{array}{cc}
S_{i}^{-1} & L_{i}^{-1} \\
* & R_{i}^{-1}
\end{array}\right] \succeq 0 .
$$

We introduce new variables

$$
J_{i}=L_{i}^{-1}, \quad U_{i}=S_{i}^{-1}, \quad H_{i}=R_{i}^{-1}
$$

so that we can re-write the condition (26) as

$$
\left[\begin{array}{cc}
U_{i} & J_{i} \\
* & H_{i}
\end{array}\right] \succeq 0
$$

This lifting provides us to use LMIs (28) and (25) instead of (22) in order to make the condition LMI.

Proposition 5.1: Consider the system (20) with each $\tilde{\Sigma}_{i}$ satisfying Lemma 5.1, if there exists a matrix $W_{i}=W_{i}^{T} \succeq$ 0 such that following LMIs hold:

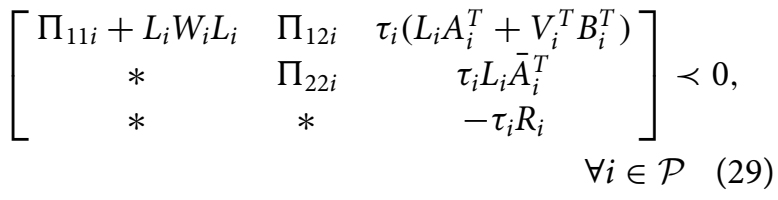

then $\dot{V}_{i}\left(t, x_{t}\right) \leq-x^{T}(t) W_{i} x(t)$ for all $i \in \mathcal{P}$.

Proof: Let us pre- and post-multiply the LMI (13) in Proposition 3.2 by $\operatorname{diag}\left[P_{i}^{-1}, P_{i}^{-1}, P_{i}^{-1}\right]$ and make the change of variables defined in Equation (23). 
Similar to the condition in Lemma 5.1, Equation (29) is also not an LMI and we handle this term with the same procedure. By defining the new variables $C_{i}$ and $O_{i}$, where $C_{i}-L_{i} W_{i} L_{i} \succeq 0$, whose Schur complement is

$$
\left[\begin{array}{ll}
C_{i} & L_{i} \\
* & O_{i}
\end{array}\right] \succeq 0
$$

and assuming $O_{i}=W_{i}^{-1}$, then we can replace the nonconvex representation in Equation (29) with $C_{i}$ as a convex one

$$
\bar{\Pi}_{i}:=\left[\begin{array}{ccc}
\Pi_{11 i}+C_{i} & \Pi_{12 i} & \tau_{i}\left(L_{i} A_{i}^{T}+V_{i}^{T} B_{i}^{T}\right) \\
* & \Pi_{22 i} & \tau_{i} L_{i} \bar{A}_{i}^{T} \\
* & * & -\tau_{i} R_{i}
\end{array}\right] \prec 0 .
$$

Now, we define lower and upper bounds for the Lyapunov functions:

$$
\begin{aligned}
& \tilde{\mu}_{i}:=\sigma_{\max }\left[L_{i}^{-1}\right]+\tau_{i} \sigma_{\max }\left[L_{i}^{-1} T_{i} L_{i}^{-1}\right]+\frac{1}{2} \tau_{i}^{2} \sigma_{\max }\left[R_{i}^{-1}\right] \\
& \tilde{\lambda}_{i}:=\sigma_{\min }\left[W_{i}\right] .
\end{aligned}
$$

Repeating the same procedure with new variables of $F_{i}$ and $E_{i},\left(F_{i} \succeq L_{i}^{-1} T_{i} L_{i}^{-1}, E_{i}:=T_{i}^{-1}\right)$,

$$
\left[\begin{array}{cc}
F_{i} & J_{i} \\
* & E_{i}
\end{array}\right] \succeq 0
$$

and re-writing $\tilde{\mu}_{i}$

$$
\tilde{\mu}_{i}=\sigma_{\max }\left[J_{i}\right]+\tau_{i} \sigma_{\max }\left[F_{i}\right]+\frac{1}{2} \tau_{i}^{2} \sigma_{\max }\left[H_{i}\right] .
$$

we obtain LMI conditions.

Consider the upper bound of the term $\tilde{\mu}_{i} / \tilde{\lambda}_{i} \leq t$. For a constant $t$, feasibility of the dwell time $\tau_{D}=t+\tau_{\max }$ is the following nonlinear SDP minimisation problem:

$$
\begin{aligned}
\min \operatorname{trace} & {\left[\sum _ { i \in \mathcal { P } } \left(L_{i} J_{i}+S_{i} U_{i}\right.\right.} \\
+ & \left.\left.R_{i} H_{i}+T_{i} E_{i}+O_{i} W_{i}\right)\right]
\end{aligned}
$$

$$
\begin{array}{rlrl}
\text { subject to } & \operatorname{diag}\left[L_{i}, T_{i}, R_{i}, Y_{i}, W_{i}\right] \succ 0, & & \forall i \in \mathcal{P}, \\
& \operatorname{diag}\left[J_{i}, F_{i}, H_{i},-W_{i}\right] & & \\
& \prec \operatorname{diag}\left[j_{i} I, f_{i} I, h_{i} I,-\tilde{\lambda}_{i} I\right], & & \forall i \in \mathcal{P}, \\
& \bar{\Lambda}_{i} \succeq 0, \bar{\Pi}_{i} \prec 0, & & \forall i \in \mathcal{P}, \\
& {\left[\begin{array}{cc}
U_{i} & J_{i} \\
* & H_{i}
\end{array}\right] \succeq 0,\left[\begin{array}{cc}
C_{i} & L_{i} \\
* & O_{i}
\end{array}\right] \succeq 0,} & \\
& {\left[\begin{array}{cc}
F_{i} & J_{i} \\
* & E_{i}
\end{array}\right] \succeq 0,} & & \\
& {\left[\begin{array}{cc}
L_{i} & I \\
* & J_{i}
\end{array}\right] \succeq 0,\left[\begin{array}{cc}
S_{i} & I \\
* & U_{i}
\end{array}\right] \succeq 0,} & \\
& {\left[\begin{array}{cc}
R_{i} & I \\
* & H_{i}
\end{array}\right] \succeq 0,} & \\
& {\left[\begin{array}{cc}
T_{i} & I \\
* & E_{i}
\end{array}\right] \succeq 0,\left[\begin{array}{cc}
O_{i} & I \\
* & W_{i}
\end{array}\right] \succeq 0,} & & \forall i \in \mathcal{P}, \\
& j_{i}+\tau_{i} f_{i}+\frac{1}{2} \tau_{i}^{2} h_{i}-t \tilde{\lambda}_{i}<0, & & \forall i \in \mathcal{P} .
\end{array}
$$

The cost function in Equation (33) is minimised to satisfy the inequality constraints (22) and (29). During the minimisation procedure, $J_{i}, U_{i}, H_{i}, O_{i}, E_{i}$ converge to $L_{i}^{-1}, S_{i}^{-1}, R_{i}^{-1}, W_{i}^{-1}, T_{i}^{-1}$, respectively. We overcome the nonlinearity of the cost function of Equation (33) by using linearisation method provided in Ghaoui, Oustry, and AitRami (1997). The linearisation of the cost function is

$$
\begin{aligned}
\underline{f_{i}=} & \text { constant }+\operatorname{trace}\left[\sum _ { i \in \mathcal { P } } \left(L_{i} J_{i}^{0}+L_{i}^{0} J_{i}+S_{i} U_{i}^{0}+S_{i}^{0} U_{i}\right.\right. \\
& \left.\left.+R_{i} H_{i}^{0}+R_{i}^{0} H_{i}+T_{i} E_{i}^{0}+T_{i}^{0} E_{i}+O_{i} W_{i}^{0}+O_{i}^{0} W_{i}\right)\right] .
\end{aligned}
$$

The linearised cost function $f_{i}$ is minimised iteratively. The cost function is re-linearised around new point $\left(L_{i}^{k}, J_{i}^{k}, S_{i}^{k}, U_{i}^{k}, R_{i}^{k}, H_{i}^{k}, T_{i}^{k}, E_{i}^{k}, O_{i}^{k}, W_{i}^{k}\right)$ in each step. The nonlinear conditions (22) and (29) are checked in each iteration up to a pre-defined number of maximum iterations. If the conditions (22), (29) and (33) are satisfied, $t$ is a proper dwell time.

The minimisation of the dwell time problem is a nested optimisation problem, where the outer loop (Steps 1 and 4) is a bisection algorithm with the cost function $\bar{f}_{i}:=t$ and the inner loop (Steps 2-4) is the optimisation problem defined in Equation (33) with the cost function $f_{i}$. If the inner loop is concluded successively, $t$ is halved in its bisection interval, otherwise doubled (Step 4).

Step 1. Choose a sufficiently large initial $t_{u}>0$ such that there exists a solution. Set $t_{l}=0$.

Step 2. Set the iteration index $k$ to 0 and $t=\left(t_{u}+t_{l}\right) / 2$. Find a feasible set for the free parameters 
$\left(L_{i}, J_{i}, S_{i}, U_{i}, R_{i}, H_{i}, T_{i}, E_{i}, O_{i}, W_{i}, Y_{i}, M_{1 i}, M_{2 i}\right.$, $\left.V_{i}, j_{i}, h_{i}, f_{i}, w_{i}\right)$ subject to conditions in Equation (33). Set $L_{i}^{0}=L_{i}, J_{i}^{0}=J_{i}, S_{i}^{0}=S_{i}, U_{i}^{0}=U_{i}$, $R_{i}^{0}=R_{i}, H_{i}^{0}=H_{i}, T_{i}^{0}=T_{i}, E_{i}^{0}=E_{i}, O_{i}^{0}=O_{i}$ and $W_{i}^{0}=W_{i}$.

Step 3. Solve the following convex optimisation problem for the same free parameters in Step 2:

$$
\begin{aligned}
\min & \operatorname{trace}\left[\sum _ { i \in \mathcal { P } } \left(L_{i} J_{i}^{k}+L_{i}^{k} J_{i}+S_{i} U_{i}^{k}+S_{i}^{k} U_{i}\right.\right. \\
& +R_{i} H_{i}^{k}+R_{i}^{k} H_{i}+T_{i} E_{i}^{k}+T_{i}^{k} E_{i} \\
& \left.\left.+O_{i} W_{i}^{k}+O_{i}^{k} W_{i}\right)\right]
\end{aligned}
$$

s.t. conditions in Equation (33).

Set $L_{i}^{k+1}=L_{i}, J_{i}^{k+1}=J_{i}, S_{i}^{k+1}=S_{i}, U_{i}^{k+1}=U_{i}$, $R_{i}^{k+1}=R_{i}, \quad H_{i}^{k+1}=H_{i}, \quad T_{i}^{k+1}=T_{i}, \quad E_{i}^{k+1}=E_{i}$, $O_{i}^{k+1}=O_{i}$ and $W_{i}^{k+1}=W_{i}$.

Step 4. If specified tolerance, such that $t_{u}-t_{l}<t o l$, is satisfied, then set $K_{i}=V_{i} L_{i}^{-1}$ for all $i \in \mathcal{P}$ and exit. The dwell time is $\tau_{D}=t+\tau_{\max }$.

Else if Equations (22) and (29) are satisfied, then set $t_{u}=t$, and return to Step 2 .

Otherwise, set $k=k+1$ and go to Step 3 . If there is no feasible solutions after specified number of iterations, then set $t_{l}=t$ and return to Step 2.

Example 5.1: This example is from Yan, Özbay, and Şansal (2014). In the corresponding paper, stabilisation of a linear time-varying system guaranteed with a switching controller. In order to achieve that, linear parameter varying (LPV) system is represented as a switching delay system with two nominal subsystems and uncertainty bounds are determined. Then, controllers are designed with robust stability conditions. Synthesised controllers are

$$
K_{1}=[0.9681,0.0465], \quad K_{2}=[-0.2708,0.3715]
$$

and the resulting dwell time is found to be 0.92 seconds. In this paper, we only considered the nominal subsystems of the switching delay system representation. The two nominal subsystems are defined as

$$
\begin{aligned}
A_{1} & =\left[\begin{array}{ll}
-3 & -1 \\
-1 & -1.9
\end{array}\right], \bar{A}_{1}=\left[\begin{array}{cc}
-1 & 0 \\
-0.45 & -1
\end{array}\right], \\
B_{1} & =\left[\begin{array}{l}
1 \\
1
\end{array}\right], \tau_{1}=0.2, d_{1}=0.01 \\
A_{2} & =\left[\begin{array}{cc}
-2 & -0.5 \\
-1 & -2
\end{array}\right], \bar{A}_{2}=\left[\begin{array}{cc}
-1 & 0 \\
0.05 & -1
\end{array}\right],
\end{aligned}
$$

$$
B_{2}=\left[\begin{array}{l}
1 \\
1
\end{array}\right], \tau_{2}=0.155, d_{2}=0.01
$$

Resulting controllers of our algorithm are

$K_{1}=[0.5527,-0.5036], \quad K_{2}=[-0.6483,-0.6561]$

and the dwell time is $\tau_{d}=0.49$ seconds.

Example 5.2: This example is a slightly modified version of the example of Yuan and Wu (2015), where the switched system in question is a non-delayed system which does not admit a common Lyapunov function. In the corresponding example, switched linear plant is in the form:

$$
\dot{x}=A_{0, \sigma(t)} x+B_{0, \sigma(t)} w+B_{1, \sigma(t)} u
$$

where $w$ is the disturbance,

$$
\begin{aligned}
A_{0,1}= & {\left[\begin{array}{ccc}
0.5108 & -0.9147 & -0.2 \\
-0.6563 & 0.1798 & 0.113 \\
0.881 & -0.7841 & 0.1
\end{array}\right], } \\
B_{1,1}= & {\left[\begin{array}{c}
0.3257 \\
1.2963 \\
2.43
\end{array}\right] } \\
A_{0,2}= & {\left[\begin{array}{ccc}
-0.125 & -0.9833 & -0.34 \\
-0.5305 & 0.3848 & 0.58 \\
1.0306 & 0.6521 & 0.1
\end{array}\right], } \\
B_{1,2}= & {\left[\begin{array}{c}
1.0992 \\
0.6532 \\
3.5
\end{array}\right] }
\end{aligned}
$$

By using $A_{0, i}$ and $B_{1, i}$, we generated our example. Let $\Sigma_{1}$ and $\Sigma_{2}$ be

$$
\begin{aligned}
A_{1} & =(1-\lambda) \cdot A_{0,1}, \quad \bar{A}_{1}=\lambda \cdot A_{0,1}, \quad B_{1}=B_{1,1}, \\
\tau_{1} & =\tilde{\tau}, \quad d_{1}=0.01, \\
A_{2} & =(1-\lambda) \cdot A_{0,2}, \quad \bar{A}_{2}=\lambda \cdot A_{0,2}, \quad B_{2}=B_{1,2}, \\
\tau_{2} & =1.6 \cdot \tilde{\tau}, \quad d_{2}=0.01 .
\end{aligned}
$$

For $\lambda=0.9$ and $\tilde{\tau}=0.05$, resulting dwell time is 13.78 seconds and controllers are

$$
\begin{aligned}
& K_{1}=[27.78,-25.94,1.70], \\
& K_{2}=[1.09,-3.08,-1.48]
\end{aligned}
$$

In Figure 2, minimum upper bounds for the dwell times can be seen for various $\tilde{\tau}$ and $\lambda$ values. Dwell time grows linearly for large delay values whereas an exponential growth is observed in Figure 1. The key difference between two examples is that the subsystems in Example 5.2 can be stabilised independent of delays but 


$$
\tau_{1}=0.05, \tau_{2}=0.08
$$

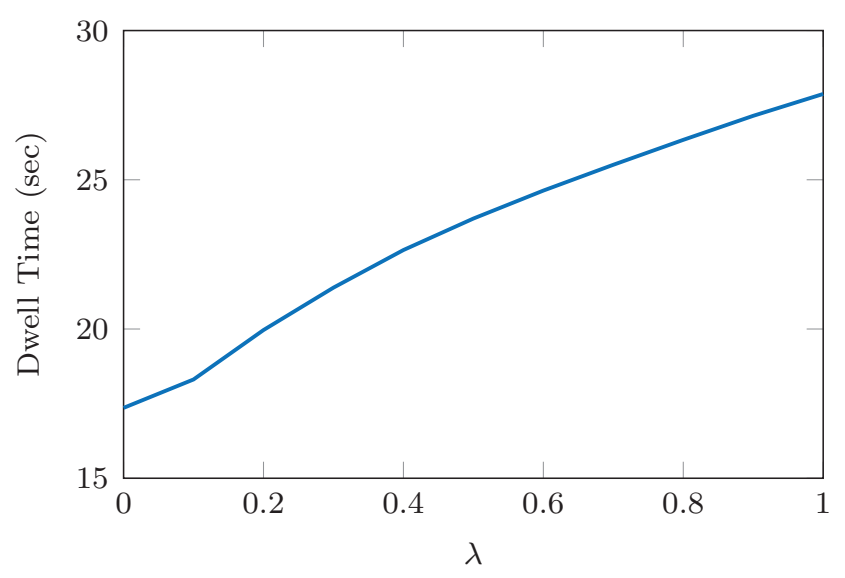

$\lambda=0.1$

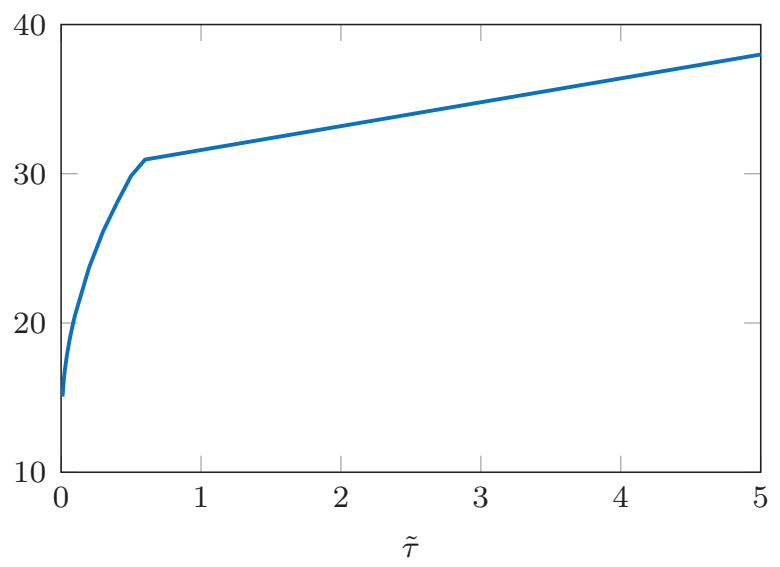

Figure 2. Dwell time results for different $\tilde{\tau}$ and $\lambda$ values of Example 5.2.

the stability of the subsystems in Example 4.1 depends on delays.

\section{Conclusions}

We performed the calculation of an upper bound of dwell time by quasi-convex optimisation methods to ensure stability of linear switched delay system. LMI conditions of free-weighting matrices method are used to find appropriate Lyapunov-Krasovskii functionals for nonswitching subsytems. By combining these conditions with a cost function, which represents the upper bound of dwell time, the upper bound is optimised using a bisection algorithm where each step is a linear SDP feasibility problem. By the numerical examples, it is shown that the results obtained in Çalışkan et al. (2013) and Yan and Özbay (2008) can be improved using the method proposed in the present paper. In addition to this, a dwell time minimising controller synthesis algorithm is also developed in this work. Although the conditions are nonlinear and the corresponding set is non-convex, this algorithm successively linearise the conditions and turn the problem into a linear SDP. The numerical examples are given to illustrate the efficiency of the proposed method.

Less conservative conditions for the stability of the delayed switching systems can be found in papers presenting average dwell time methods (see Sun et al., 2006; Chesi, Colaneri, Geromel, Middleton, \& Shorten, 2012). However, the average dwell time conditions are nonconvex due to exponential and bilinear terms when the dwell time is considered as a free parameter in optimisation. Representation of the dwell time in the present paper is more conservative, but the dwell time minimiser controller synthesisation problem is tractable due to convex nature of the conditions.
A typical application of the switched control scheme is the network congestion control systems (Zhao, Zhang, Shi, \& Liu, 2012). Due to the time-delay nature of the network systems, the method presented in this paper can contribute to the research on application of the network congestion control systems.

\section{Disclosure statement}

No potential conflict of interest was reported by the authors.

\section{ORCID}

Ahmet Taha Koru (ㄱ) http://orcid.org/0000-0001-8191-2324

\section{References}

Allerhand, L., \& Shaked, U. (2011). Robust stability and stabilization of linear switched systems with dwell time. IEEE Transactions on Automatic Control, 56(2), 381-386.

Boyd, S., \& Vandenberghe, L. (2004). Convex optimization. Cambridge: Cambridge University Press.

Bullo, F., \& Liberzon, D. (2006). Quantized control via locational optimization. IEEE Transactions on Automatic Control, 51(1), 2-13.

Çalışkan, S.Y., Özbay, H., \& Niculescu, S.I. (2013). Dwell-time computation for stability of switched systems with time delays. IET Control Theory \& Applications, 7(10), 14221428.

Chen, W.H., \& Zheng, W.X. (2010). Delay-independent minimum dwell time for exponential stability of uncertain switched delay systems. IEEE Transactions on Automatic Control, 55(10), 2406-2413.

Chesi, G., Colaneri, P., Geromel, J.C., Middleton, R., \& Shorten, R. (2012). A nonconservative LMI Condition for stability of switched systems with guaranteed dwell time. IEEE Transactions on Automatic Control, 57(5), 1297-1302.

Fainshil, L., Margaliot, M., \& Chigansky, P. (2009). On the stability of positive linear switched systems under arbitrary 
switching laws. IEEE Transactions on Automatic Control, 54(4), 897-899.

Geromel, J.C., \& Colaneri, P. (2006). Stability and stabilization of continuous time switched linear systems. SIAM Journal on Control and Optimization, 45(5), 1915-1930.

Ghaoui, L.E., Oustry, F., \& AitRami, M. (1997). A cone complementarity linearization algorithm for static outputfeedback and related problems. IEEE Transactions on Automatic Control, 42(8), 1171-1176.

$\mathrm{Gu}, \mathrm{K} .$, Kharitonov, V., \& Chen, J. (2003). Stability of time-delay systems. Boston, MA: Birkhauser.

He, Y., Wang, Q.G., Xie, L., \& Lin, C. (2007). Further improvement of free-weighting matrices technique for systems with time-varying delay. IEEE Transactions on Automatic Control, 52(2), 293-299.

Hespanha, J.P. (2004). Uniform stability of switched linear systems: Extensions of LaSalle's invariance principle. IEEE Transactions on Automatic Control, 49(4), 470482.

Hespanha, J.P., \& Morse, A.S. (1999). Stability of switched systems with average dwell-time. In Proceedings of the 38th IEEE Conference on Decision and Control (Vol. 3, pp. 26552660), Phoenix, AZ: IEEE.

Hou, M., Fu, F., \& Duan, G. (2013). Global stabilization of switched stochastic nonlinear systems in strict-feedback form under arbitrary switchings. Automatica, 49(8), 25712575.

Ishii, H., \& Francis, B.A. (2001). Stabilizing a linear system by switching control with dwell time. In Proceedings of the American Control Conference (Vol. 3, pp. 1876-1881), Arlington, VA: IEEE.

Koru, A.T., Delibaşı, A., \& Özbay, H. (2014). On dwell time minimization for switched delay systems: Free-weighting matrices method. In Proceedings of the 53rd IEEE Conference on Decision and Control (pp. 1978-1982). Los Angeles, CA: IEEE.

Li, Z., Gao, H., Agarwal, R., \& Kaynak, O. (2013). $H_{\infty}$ control of switched delayed systems with average dwell time. International Journal of Control, 86(12), 2146-2158.

Liberzon, D., \& Morse, A.S. (1999). Basic problems in stability and design of switched systems. IEEE Control Systems, 19(5), 59-70.

Lin, H., \& Antsaklis, P.J. (2009). Stability and stabilizability of switched linear systems: A survey of recent results. IEEE Transactions on Automatic Control, 54(2), 308-322.
Mahmoud, M.S. (2010). Switched time-delay systems. Boston, MA: Springer-Verlag.

Mitra, S., \& Liberzon, D. (2004). Stability of hybrid automata with average dwell time: An invariant approach. In $43 \mathrm{rd}$ IEEE Conference on Decision and Control (Vol. 2, pp. 13941399), Atlantis, Paradise Island, Bahamas: IEEE.

Morse, A.S. (1996). Supervisory control of families of linear setpoint controllers Part I. Exact matching. IEEE Transactions on Automatic Control, 41(10), 1413-1431.

Shorten, R., Narendra, K., \& Mason, O. (2003). A result on common quadratic Lyapunov functions. IEEE Transactions on Automatic Control, 48(1), 110-113.

Sturm, J.F. (1999). Using SeDuMi 1.02, a Matlab toolbox for optimization over symmetric cones. Optimization Methods and Software, 11(1-4), 625-653.

Sun, X.M., Zhao, J., \& Hill, D.J. (2006). Stability and $L_{2}$-gain analysis for switched delay systems: A delay-dependent method. Automatica, 42(10), 1769-1774.

Sun, Z., \& Ge, S. (2011). Stability theory of switched dynamical systems. London: Springer-Verlag.

Wu, M., He, Y., \& She, J.H. (2010). Stability analysis and robust control of time-delay systems. London: Science Press Beijing and Springer-Verlag.

Yan, P., \& Özbay, H. (2008). Stability analysis of switched time delay systems. SIAM Journal on Control and Optimization, 47(2), 936-949.

Yan, P., Özbay, H., \& Şansal, M. (2014). Robust stabilization of parameter varying time delay systems by swiched controllers. Applied and Computational Mathematics, 13(1), 31-45.

Yan, P., Özbay, H., \& Sansal, M. (2011). Dwell time optimization in switching control of parameter varying time delay systems. In 50th IEEE Conference on Decision and Control and European Control Conference (CDC-ECC) (pp. 49094914), Orlando, FL: IEEE.

Yuan, C., \& Wu, F. (2015). Hybrid control for switched linear systems with average dwell time. IEEE Transactions on Automatic Control, 60(1), 240-245.

Zhang, J., Han, Z., Zhu, F., \& Huang, J. (2013). Stability and stabilization of positive switched systems with modedependent average dwell time. Nonlinear Analysis: Hybrid Systems, 9, 42-55.

Zhao, X., Zhang, L., Shi, P., \& Liu, M. (2012). Stability of switched positive linear systems with average dwell time switching. Automatica, 48(6), 1132-1137. 